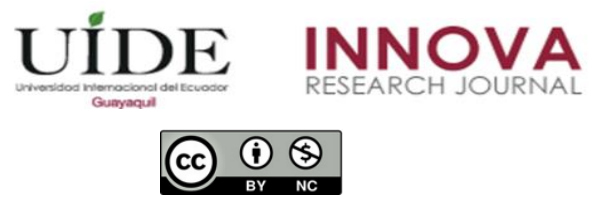

INNOVA Research Journal, ISSN 2477-9024

(Septiembre-Diciembre 2020). Vol. 5, No.3 pp. 122-134

DOI: https://doi.org/10.33890/innova.v5.n3.2020.1358

URL: http://revistas.uide.edu.ec/index.php/innova/index

Correo: innova@uide.edu.ec

\title{
Optimización en la Gestión de Registro y Control de Fichas Estudiantiles del Departamento de Bienestar Estudiantil
}

\section{Optimization in the management of registration and control of student records of the Department of Student Welfare}

Giovanni M. Rizzo Vargas

Investigador independiente, Ecuador

Alejandra M. Colina Vargas

https://orcid.org/0000-0003-1514-8852

Universidad ECOTEC, Ecuador

José J. Túa Ollarves

https://orcid.org/0000-0001-5054-5920

Universidad Nacional Experimental Francisco de Miranda (UNEFM), Venezuela

Autor para correspondencia: barcegiorizzo@gmail.com; giovanni_rizzo360@ hotmail.com; acolina@ecotec.edu.ec; jah120@gmail.com

Fecha de recepción: 22 de febrero del 2020 - Fecha de aceptación: 10 de junio del 2020

\section{Resumen}

El artículo pretende mostrar como a partir del desarrollo de una solución tecnológica dirigida al departamento bienestar estudiantil de una universidad del Ecuador se optimizan de forma eficiente los procesos de gestión en el registro y control de las fichas estudiantiles para la atención a los estudiantes de nuevo ingreso a la universidad. Para ello se llevó a cabo se articulación de las metodologías de investigación de tipo exploratoria y descriptiva apoyada con herramientas y técnicas del desarrollo ágil de software. La solución propuesta fue desarrollada bajo estándares de programación estrictamente bien elaborados, basados en herramientas de software libre que mejoran y favorecen una actualización constante de la arquitectura del sistema. Se implementó para la construcción la metodología SCRUM identificando la visión general del producto a partir del diagnóstico in situ y la revisión documental, posteriormente se listan de forma priorizada los requisitos y objetivos que representan la visión y expectativas del cliente siendo cubiertos de la mejor manera posible. Destaca finalmente, dentro los resultados un nivel alto de satisfacción por parte de los usuarios por considerarse un producto de utilidad que favorece el área de bienestar estudiantil mejorando el proceso y registro de la información acumulativa de cada estudiante y el seguimiento adecuado del mismo.

Palabras clave: optimización; solución tecnológica; bienestar estudiantil; universidad; metodologías ágiles 


\begin{abstract}
The article aims to show how, from the development of a technological solution directed to the student welfare department of a university in Ecuador, the management processes in the registration and control of student records are efficiently optimized for the care of new students. to the University. To this end, an articulation of exploratory and descriptive research methodologies was carried out, supported by tools and techniques of agile software development. The proposed solution was developed under strictly well-developed programming standards, based on free software tools that improve and favor a constant update of the system architecture. The SCRUM methodology was implemented for the construction identifying the general vision of the product from the on-site diagnosis and the documentary review, later the requirements and objectives that represent the vision and expectations of the client are listed as a priority, being covered in the best possible way. Finally, within the results, a high level of user satisfaction stands out as it is considered a useful product that favors the area of student well-being by improving the process and recording of the cumulative information of each student and the adequate follow-up of the same.
\end{abstract}

Key words: optimization; technological solution; student welfare; university; agile methodologies

\title{
Introducción
}

En la actualidad, el potencial humano y la innovación son referentes considerados por las organizaciones para apostar a alcanzar ventajas competitivas, emergiendo entre ellos la tecnología y sus distintas formas de incorporación en las estrategias organizacionales como soporte y apoyo en la promoción e innovación de procesos, productos, servicios y sistemas, que añaden a los progresos existentes. En palabras de (Mosqueda \& Millán, 2013) cada uno los procesos y operaciones que se llevan a cabo en las organizaciones llevan consigo transformación, incorpora suministros y manipula materias primas, que son convertidos posteriormente en bienes y/o servicios que los consumidores demandan.

Las organizaciones incorporan hoy la tecnología no únicamente para aumentar la infraestructura tecnológica, al contrario, comprende mecanismo que favorecen la generación de bienes o servicios de calidad, con precio ajustado a lo que el mercado que demande. Enfrentándose, entonces, al hecho de que deben realizar estudios preliminares que les permitan reconocer qué tecnologías pueden apoyarse para cumplir los objetivos de estratégicos; quién será el proveedor; y finalmente cómo llevar a cabo el acompañamiento para una implementación efectiva, que involucre la protección de los desarrollos tecnológicos propios (Solleiro \& Castañón, 2016).

La irrupción en las organizaciones de las tecnologías de información con sus avances e innovación ha favorecido una dinámica de vida diferente, convirtiendo el conocimiento en ese bien intangible valioso. Con ello se ha afectado la realización de tareas rutinarias en todos los sectores de la sociedad, destacándose entre ellos la medicina, las telecomunicaciones, la economía y la propia educación. Este escenario conlleva a dar respuesta a nuevos retos y desafíos, que los obligan a replantear como enfrentar un entorno dinámico y crear nuevas alternativas para satisfacer las necesidades de cada organización en la llamada sociedad del conocimiento. (Gómez O., Lona R., \& Jiménez S., 2016). 
Para dar respuesta al contexto referenciado, el sector de la educación debe innovar para no perecer y responder de manera efectiva y continua a las demandas y necesidades de una sociedad que requiere la formación de profesionales preparados para enfrentar nuevos problemas sociales; conlleva este escenario la necesidad de que las universidades estén actualizadas, requiere para ello re-plantear y re-formular los objetivos institucionales, procesos, modelos educativos, entre otros (Vázquez P. \& Ortiz M., 2018). Este sector o nivel de la educación constituye para el país un mecanismo por el cual se aspira alcanzar el progreso, bienestar y desarrollo de la sociedad y la economía, permitiendo así la igualdad de oportunidades para la población y un ascenso social en la comunidad en general (Salcedo-Muñoz, y otros, 2017).

Para las universidades, la tecnología juega un papel cada vez más preponderante. Se requiere una reorientación profunda, seria y estratégicamente planificada de la gestión de la tecnología. Siendo evidente hoy la clara dependencia de la tecnología en todos los procesos universitarios (García H. \& García P., 2015). La universidad no es y no puede ser ajena a esta integración en el mundo digital, ellas están llamadas a rendir cuenta a la sociedad como cualquier otra organización.

Sobre estos argumentos, los modelos de funcionamientos de las organizaciones se encuentran más ligado que nunca al uso de la tecnología, al alcance y efectos que puede tener a nivel comunicativo y de desempeño en general (Grande, Cañón, \& Cantón, 2016). Existiendo, entonces una correlación evidente entre la tecnología y la eficacia con la que pueda responder en sus procesos, lo cual no es nada fácil de planificar sobre todo en instituciones con muchos años de historia y con una idiosincrasia muy peculiar. Es por ello que, la gestión de la tecnología se convierte en uno de los grandes retos de la universidad en esta década, pues ya está inmersa en los procesos universitarios (Solleiro \& Castañón, 2016).

Todas las organizaciones en la actualidad generan una gran cantidad de información, tanto en sus procesos internos como en sus procesos externos. De allí, la importancia que tiene una adecuada gestión de la información dado el valor que representa para las mismas, el cual ha sido estudiado desde la antigüedad hasta el presente; gestión que hoy es soportada por lo que se conoce como sistemas de información (Posner, 1972) (García H. \& García P., 2015).

Se requiere entonces, en palabras de (Rodríguez S. \& Colina V., 2018) que las universidades incorporen las tecnologías para favorecer los niveles de acceso, registro y control de información de calidad como apoyo al cumplimiento de objetivos estratégicos y la consecución de procesos eficiente y eficaces.

La Universidad Tecnológica ECOTEC, en los últimos años ha formulado un conjunto de políticas estratégicas, las cuales definen las directrices generales que norman los procesos en la institución, entre ellas cabe mencionar las políticas de calidad comprometidas con la consolidación de procesos sustantivos (docencia, investigación y vinculación) y de apoyo alineados a una continua mejora, apuntando al uso e incorporación de tecnología y sistemas de información que favorezcan el desarrollo institucional (ECOTEC, Políticas Institucionales, 2019) 
Para operacionalizar este objetivo estratégico se ha dado un avance significativo en lo que corresponde a la infraestructura tecnológica, con proyectos de implementación de diversos sistemas de información que contribuyen a la automatización de los procesos concernientes a la universidad en los últimos años, que van desde lo académico hasta la gestión administrativa en particular en el área de Bienestar Estudiantil y el Servicio Médico.

En la Ley orgánica de Educación Superior se establece que, las instituciones de educación superior mantendrán una unidad administrativa de bienestar destinada a promover los derechos de los distintos estamentos de la comunidad académica, y desarrollará procesos de orientación vocacional y profesional, además de obtención de créditos, estímulos, ayudas económicas y becas, y ofrecerá servicios asistenciales que se determinen en las normativas de cada institución (Ley Orgánica De Educación Superior, 2010).

Destaca entonces de manera significativa, el rol que desempeñan estas unidades administrativas en las universidades como subsistema de soporte en la formación académica, de ayuda económica y de adhesión social de los estudiantes, desarrolla, ejecuta programas y servicios con el propósito de ofrecer una atención individualizada que facilite la atención de las necesidades académicas, físicas y psicológicas de los estudiantes.

La Universidad Tecnológica ECOTEC, por su parte, establece como objetivo estratégico "garantizar las condiciones propicias en el desarrollo de un entorno educativo, científico y cultural adecuado para la formación integral de estudiantes y graduados comprometidos con el progreso y desarrollo social" (ECOTEC, Plan de Desarrollo Estratégico Institucional 2017 2021, 2017).

Dentro de la estructura de apoyo se dispone en la Universidad Tecnológica ECOTEC de la unidad o departamento de bienestar estudiantil conformado por un equipo multidisciplinario de profesionales con la finalidad de orientar y asesorar a los estudiantes durante su formación profesional. Esta dependencia ofrece alternativas de solución a situaciones socio-familiares, socio-económicos y otras dificultades que el estudiante puede sortear durante sus estudios universitarios proporcionando bienestar personal y familiar.

Diversas instituciones educativas en el país se encuentran en busca de la excelencia académica y por consiguiente la gestión de los procesos que se desarrollan dentro de ellas, para esto se hace uso de sistemas de información que facilitan y ayudan en los procesos académicos y administrativos que se llevan en ella, así mismo deben dar respuesta al aumento de aspirantes a las diversas carreras, por tal motivo las universidades replantean sus procesos a fin de dar respuesta a lo antes mencionado.

Es de interés en este artículo abordar como objeto de investigación la dirección de bienestar estudiantil, la cual cada día se ven más limitadas en el proceso de captura de datos de los estudiantes nuevos haciendo referencia a tareas repetitivas como las de registrar, almacenar, reportar la información, la cual logra saturar las actividades y genera pérdida de recursos dentro del departamento. En esta dirección, es necesario la evaluación de los estudiantes que ingresan a los primeros semestres de la universidad, los cuales permitirá obtener una mejor apreciación de las carreras de acuerdo a las aptitudes y habilidades vocacionales, para la elaboración correcta de 
la ficha que permita a la responsable del área sugerir y acompañar al estudiante de la opción de carrera universitaria. Esta dependencia es la encargada de realizar todas las tareas de registro y control de fichas de información.

El proceso de captura de información de las fichas acumulativas de los estudiantes según el responsable del área no cuenta con una solución tecnológica que permita optimizar el tiempo de entrega de resultado de dicha información, esto debido a que la mayor parte de los test son elaborados de forma manual, lo cual genera una acumulación de procesos en el análisis de resultados de la información y demora en la entrega de reportes.

Para ello, se realizó un diagnóstico en el departamento antes mencionado a través de entrevistas a la directora de dicha entidad, expresando la existencia de ciertas necesidades relacionadas con el registro de las fichas acumulativas de información de los estudiantes: Test de Inteligencias Múltiples, Test de Interés Vocacional y Profesional, Test de Interés.

Es una aspiración de este articulo plantear como alternativa de solución al problema señalado el desarrollo e implementación de una solución tecnológica a fin de poder obtener mejoras en los procesos de una manera adecuada validando la información sin temor a obviar algún dato importante del alumno, así mismo facilitará y optimizará los tiempos de respuesta para las fichas acumulativas de información y la obtención de los reportes.

Con el desarrollo de la investigación se optimizará de forma más eficiente la realización de la tarea de registrar datos en las fichas estudiantiles al ingresar de manera digital, liberando la forma tradicional de hacerlo y evitando los errores que suelen cometer los responsables de la dirección al momento de acentuar los datos a la ficha. Así mismo, representa avances significativos de implementación nuevas tecnología que facilite el trabajo que se realiza a diario en dicho departamento (Alvia Choez \& Vera Macías, 2018).

Por lo tanto, el presente artículo tiene como objetivo brindar soporte a través del desarrollo de una solución tecnológica que permita resolver las necesidades y requerimientos encontrados en el departamento de bienestar estudiantil de la universidad ECOTEC, entre los que destacan: falencias en la captura de información de las fichas acumulativas, retraso en la entrega de resultados, entre otros. Para el diseño de la solución tecnológica propuesta se utilizó una arquitectura y estándares bajo el soporte de herramientas de software libre para la creación de diseños, estadísticas y reportes, el cual este integrado con el sitio web de la universidad ECOTEC.

\section{Revisión teórica}

\section{Sobre el proceso del Departamento de Bienestar}

La gestión de procesos facilita en la organización la identificación, diseño, control, mejorar de procesos más productivos y en esa medida favorece el clima de confianza del cliente $\mathrm{y}$ aumentar la productividad en un entorno con grandes desafíos en tiempos de exigencias crecientes (Bravo C., 2011). 
La dirección de bienestar estudiantil procura la mejora en la calidad de vida de los principales actores de una universidad, es decir el estudiante, para ello pretende promover y garantizar la estancia en ese sistema educativo de los estudiantes, pues tanto el estado ecuatoriana como las empresas particulares hacen inversión social en favor de la sociedad en general (Salcedo-Muñoz, y otros, 2017).

A los fines de la investigación se partirá de un enfoque de procesos como forma de ver la totalidad la Dirección de Bienestar Estudiantil de la Universidad ECOTEC procurando la igualdad de oportunidades para el desarrollo personal de los estudiantes en los ámbitos sociales y culturales con el fin de lograr un mejoramiento integral de la calidad de vida de los estudiantes promoviendo la orientación vocacional y profesional en un ambiente de respeto a los derechos de los estudiantes con fraternidad, cooperación y superación profesional y cultural (Resolución Nro. 27-14-09-2016., 2016). Las normas y reglamentos vigentes que rigen el actuar de esta dirección comprenden la Ley orgánica de educación, el Reglamento de Bienestar Estudiantil, Admisiones y Graduados y el Plan Estratégico de Desarrollo Institucional vigente de la Universidad ECOTEC.

En los departamentos de bienestar estudiantil se establecen programas, proyectos y servicios centrados en atender la salud física y mental, temas deportivos y culturales, planes preventivos de salud; así como también un conjunto de acciones que favorezcan el proceso de formación estudiantil, estableciendo un eje integral y social (Salcedo-Muñoz, y otros, 2017).

Dentro de los servicios o programas que dispone la dirección de Bienestar Estudiantil de la Universidad ECOTEC de acuerdo al reglamento vigente en su Artículo 10 está ser guía vocacional y profesional promoviendo actividades de seguimiento psicológica, a que permita "identificar por medio de la evaluación psicológica las tendencias vocacionales de sus miembros" (Resolución Nro. 27-14-09-2016., 2016).

Este servicio lleva consigo un registro y control de las fichas acumulativas de los estudiantes que ingresan a la Universidad, para lo cual se lleva a cabo los procesos de: Orientación Vocacional y Consejería Psicológica, Gestión de Becas y Ayudas Económicas, Seguimiento (Inserción Laboral), Derecho e Integridad (Planificación) y Servicios Asistenciales. De los procesos antes mencionados fue necesario a los fines de la presente investigación el rediseño del proceso de Orientación Vocacional y Consejería a fin de ayudar a la Dirección de Bienestar Estudiantil en la forma de enfocar el cambio y optimizar los procesos (Bravo C., 2011), cubriendo los siguientes: Test: Orientación Vocacional, Inteligencia Múltiple, Personalidad, Ingreso de Ficha, Asesoría y Seguimiento.

\section{En relación a la metodología de desarrollo SCRUM}

El enfoque utilizado para la elaboración de la propuesta de optimización en este artículo será de métodos ágiles caracterizado por la participación directa en todas las fases del cliente o usuario final, estableciendo lineamiento de diseño rápido, ofreciendo liberaciones continuas del prototipo, en este sentido se reducen gastos y se alcanza una producción de código de alta calidad (Sommerville, 2011). 
Teniendo entre sus referentes la metodología de desarrollo Scrum considerada como un esquema de trabajo ligero que facilita el desarrollo e problemas complejos, con entregas de productos de forma eficiente y creativa con el máximo valor. Permite emplear varios procesos y técnicas de gestión de producto para mejorar continuamente el producto, el equipo y el entorno de trabajo (Schwaber \& Sutherland, 2017). Para ello, se establecen ciclos breves para el desarrollo, que comúnmente eran conocidos como interacciones y en esta metodología adoptan la connotación de "sprints" (Trigas G., 2012).

Esta metodología ágil se caracteriza por facilitar un esquema de trabajo para la administración del proyecto. Utilizando los "sprints", como espacios periódicos que muestran el desarrollo en forma incremental del sistema. Para ello se elabora una planeación basada en dar prioridad a los trabajos atrasados y seleccionar las tareas de importancia más alta para un sprint.

Las fases que comprende la metodología de Scrum según (Sommerville, 2011) son tres: Primera fase, la planeación del bosquejo, definición de los propósitos generales del proyecto y propuesta de arquitectura del software. Fase segunda, ejecución de ciclos sprint, elaboración incremental del sistema. Por último, el cierre del proyecto se finiquita el proyecto y toda la documentación correspondiente, marcos de ayuda del sistema y manuales del usuario, valorando las enseñanzas asimiladas en el proyecto (Figura 1).

\section{Figura 1.}

Fases del proceso de Scrum

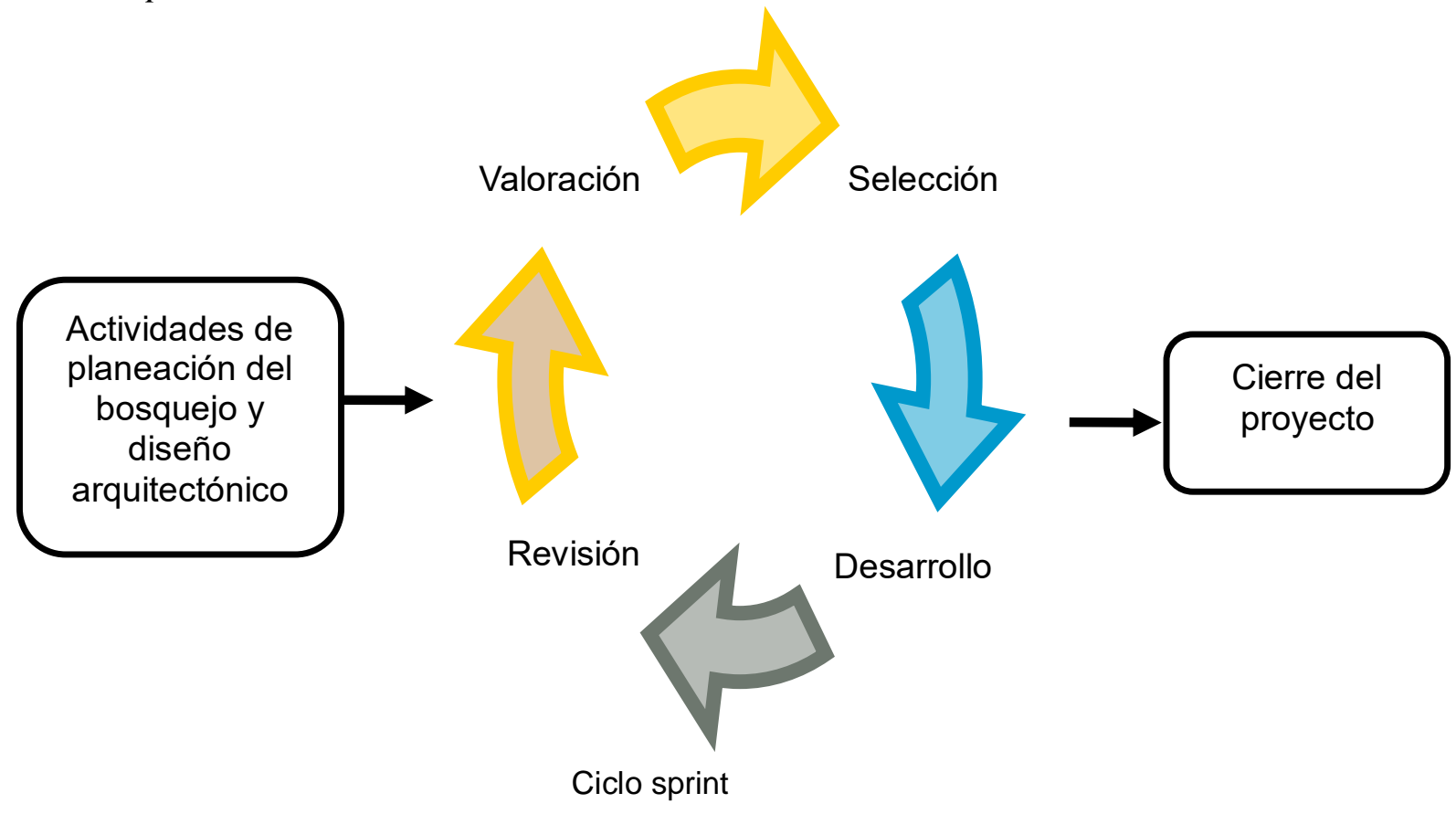

Fuente: Adaptado de Sommerville (2011)

La fase central del proceso de Scrum lleva la ejecución de ciclos de "sprints". Estos ciclos se planean como espacio que evalúa el trabajo que se va a realizar, tomando en cuenta las particularidades pendientes de desarrollar e implementar. Una vez completado los sprint, se hace llegar a los participan el software (Sommerville, 2011). 
La metodología Scrum va dirigida principalmente tanto a las personas como el equipo de desarrollo que participan en la elaboración del producto. Plantea un trabajo unificado entre los miembros del equipo a fin de obtener productos complejos y sofisticados eficientes (Trigas G., 2012). Comprende la definición de un equipo de trabajo, la definición de roles, de eventos, de artefactos que integran el marco de trabajo que contribuye al éxito del proyecto (Schwaber \& Sutherland, 2017).

\section{Artefactos de Scrum}

Los artefactos o aparatos de Scrum constituyen las distintas formas que garantizan la transparencia y oportunidades para la inspección y adaptación de la información clave, necesaria para confirmar que todos tengan la comprensión unificada del artefacto.

\section{Partes de Scrum}

Dentro de las partes generales que constituyen scrum están el conjunto de fases y roles. Se siguen una serie de encuentros a fin de planear, ejecutar, seguir y revisar los sprints (Trigas G., 2012) destaca como roles: producto owner, scrum master, equipo de trabajo, interesados y administradores (Tabla 1).

\section{Tabla 1.}

Papel de actuación en la metodología de Scrum.

\begin{tabular}{ll}
\hline Papel de actuación & Definición \\
\hline Product owner & delegado de tomar de decisiones. \\
Scrum master & responsable de la aplicación correcta del modelo y la metodología \\
Equipo de trabajo & grupo de personas que ejecutan las necesidades del cliente. \\
Interesados & Serán beneficiados de proyecto, participan en la revisión del sprint. \\
Administradores & Tomadores de decisiones sobre los requisitos y objetivos del producto. \\
\hline
\end{tabular}

Fuente: elaboración propia

\section{Componentes de Scrum}

Los componentes que integran scrum están: 1.- Product backlog: lista de necesidades del cliente; 2.- Sprint backlog: lista de tareas que se realizan en un sprint; 3.- Incremento: parte terminada y totalmente operativa que se añade o se desarrolla en un sprint (Trigas G., 2012).

\section{Conjunto de Producto (Product Backlog)}

El conjunto de requisitos del producto representa un ordenado listado de los elementos que se conoce, aquellos que son necesario en el producto. Concentra el conjunto de tareas, requerimientos y funcionalidades imprescindibles. El Dueño de Producto (Product Owner) es la única autoridad para agregar, actualizar y priorizar su contenido. 


\section{Metodología}

Esta investigación se caracterizó por ser descriptiva, se estudió el proceso general del departamento de bienestar estudiantil a fin de obtener detalle de los procesos y actividades que deben considerarse en la solución tecnológica propuesta. Así mismo, tipo de investigación aplicada, pues parte de los conocimientos adquiridos, así como también se emplearon diversas fuentes referidas al desarrollo de soluciones tecnológicas en las organizaciones, con la finalidad de optimizar sus procesos y en esa medida incrementar la productividad.

\section{Ciclo de desarrollo de la metodología Scrum}

La metodología de Scrum inicia como todas las metodologías de desarrollo ágiles conociendo las necesidades de los clientes, sin embargo, no centra los esfuerzos en requerimientos formales predefinidos, al contrario, plantea involucrar al cliente como parte del equipo de desarrollo. Asimismo, el desarrollo del proyecto gira en torno a unos requisitos que emergen implícitamente dentro del contenido de las denominadas historias de usuario. Acá es donde prevalece la necesidad de entrega más rápida del software, que es más adecuada para las necesidades del cliente, se aplica también a sistemas más grandes (Sommerville, 2011).

De esta manera, los requisitos se dividen en entregas parciales o fases, en función de las prioridades y conforme al valor aportado un equipo de auto-organización en incrementos (llamados "sprints"), empezando por la planificación y finalizando con un comentario. Los períodos de trabajo suelen estar entre 2 y 4 semanas, para consignar algo que funcione y vaya evolucionando de acuerdo a las pruebas de usuario (owner), de modo que se puedan introducir los cambios necesarios, antes de que sea demasiado tarde.

Para ello, se registra un Backlog o documento descriptivo de tareas a entregar en la finalización de los sprints, junto con las horas de trabajo, responsables y costos, asumiendo un diagrama burn down o diagrama de quemado, marcando el estado y la evolución, indicando las tareas y requerimientos pendientes de ser tratados a lo largo del tiempo. De una forma, intuitiva se tiene que todo transcurre según lo previsto o existe alguna desviación de acuerdo a la fecha de entrega.

Parte del equipo de trabajo (Scrum Team) coordinan su trabajo en un diario de stand-up de la reunión (sprint planning meeting), cuyo lapso de tiempo no ha de exceder los 15 minutos. Un miembro del equipo, el "Scrum Master" (equivalente al gerente del proyecto), ha de encargarse de orientar las reuniones pivoteando en encontrar respuestas a ¿qué has hecho desde ayer?, ¿qué tienes pensado hacer hasta mañana?, y ¿has conseguido problemas para alcanzar tu objetivo?

\section{Resultados}

\section{Ejecución de la metodología scrum}

Tomando en consideración la metodología descrita se siguió las fases o etapas de Scrum: 


\section{Planificación del Backlog}

La primera fase, elaboración del plan comprendió el listado con los requisitos del producto llamado producto backlog, mantenido y ordenados por prioridades por el Product Owner, construyéndose un documento dinámico que incorporó constantemente las necesidades del sistema, para ello se llevaron a cabo encuentros o Sprint con responsables del área de Bienestar Estudiantil. A partir del cual se levantan las primeras necesidades. Las Tabla 2 y Tabla 3 muestran los resultados del levantamiento de requisitos.

Tabla 2.

Product backlog o requisitos de la solución propuesta.

\begin{tabular}{|c|c|}
\hline Tipo de Requisitos & Descripción \\
\hline Funcionales & $\begin{array}{l}\text { - } \text { Selección de estudiante mediante base de datos académica } \\
\text { - } \text { Almacenamiento de información a través de sistema de base de datos } \\
\text { - } \quad \text { Tabulación de datos, mediante resultados obtenidos } \\
\text { - } \quad \text { Emisión de reportes de acuerdo a las características solicitadas } \\
\text { - Ingresar y consultar datos de los estudiantes. }\end{array}$ \\
\hline No funcionales & $\begin{array}{l}\text { - } \text { Sistema compatible con distintos navegadores web } \\
\text { - } \text { Interfaz de fácil usabilidad para el usuario } \\
\text { - } \text { Motor de base de datos de uso múltiple y adaptable a cambios } \\
\text { - } \quad \text { Tiempos oportunos de respuesta } \\
\text { - } \quad \text { Sistema de fácil operatividad } \\
\text { - Acceso a administrador y estudiantes por medio de base de datos académica } \\
\text { - Almacenamiento de información en línea } \\
\text { - } \quad \text { Disponibilidad del sistema } 24 / 7\end{array}$ \\
\hline
\end{tabular}

Fuente: elaboración propia.

Tabla 3.

Otros product backlog de la solución propuesta

\begin{tabular}{ll}
\hline Backlog o requisitos técnicos & Descripción \\
\hline Programa del sistema & $\begin{array}{l}\text { Cualquier sistema operativo que soporte Google Chrome y Mozilla } \\
\text { Firefox }\end{array}$ \\
Explorador web & $\begin{array}{l}\text { Google Chrome (todas sus versiones), Mozilla Firefox (todas sus } \\
\text { versiones). }\end{array}$ \\
Software de gestión de base de & Microsoft SQL Server 2016 \\
datos & PHP Framework (Codeigniter 3.1.5), HTML,CSS, JS Framework \\
Lenguajes de programación & (Bootstrap 2.8) \\
\hline
\end{tabular}

Fuente: elaboración propia.

\section{Seguimiento del Sprint:}

Para ir evaluando el desarrollo en cada una de sus fases se comprobó por día el desempeño de los requerimientos del proyecto, a fin de seguir con el Sprint se formulaban subsecuentemente las tareas realizadas. Durante la revisión del trabajo ejecutado en los primeros Sprint fue presentados a los responsables del Departamentos de Bienestar Estudiantil los diagramas de casos de usos, para validar los requisitos levantados al inicio. 


\section{Revisión del Sprint:}

Las actividades del proyecto se llevaron a cabo por un periodo de 35 días.

\section{Incremento}

La fase de incremento describe el nivel de avance de los Sprint hasta culminar los requisitos del Product Backlog en su totalidad, se completaron siete sprint con un avance incremental de $13,87 \%$ en el primero, el segundo de $28,225 \%$, el tercero de $42,58 \%$, cuarto de $56,935 \%$, quinto de $71,29 \%$, sexto de $85,645 \%$ hasta llegar en el séptimo al $100 \%$, posteriormente se realizó la entrega total del proyecto.

\section{Validación de la propuesta tecnológica}

La última fase comprendió las actividades centradas en validar la conformidad de los requerimientos e impacto para el área de bienestar estudiantil con la puesta en marcha de la solución propuesta y en esa medida valorar el impacto en la optimización de los procesos de registro y control de fichas estudiantiles en dicho departamento. Los criterios considerados en la validación fueron: interfaz, contenido, sesión de inicio, usabilidad, flexibilidad.

\section{Análisis de Resultados}

De los resultados obtenidos se logró comprobar que los tiempos resultantes tanto al ingresar datos como mostrar resultados son óptimos, sin embargo, dependiendo del volumen de datos que se ingresan los tiempos pueden variar. Es importante, considerar que dependiendo de la conexión de ancho de banda favorecerá la generación reportes o ingreso de información, pues el sistema está enlazado directamente con el servidor de la universidad ECOTEC, lo cual ayuda a reducir tiempos de respuesta logrando un sistema de calidad.

Durante las diferentes pruebas realizadas se tomaron en consideración varios criterios con los cuales se realizó una tabla comparativa (Tabla 4) en las formas y el tiempo de demora, en el cual se ejecutaban los procesos anteriormente y ahora que se han optimizado con el sistema de registro y control.

\section{Tabla 4.}

\section{Comparativa de demostración}

\begin{tabular}{|c|c|c|}
\hline Criterios & Antes & Ahora \\
\hline Portabilidad & $\begin{array}{l}\text { Baja.- solo existe sistematizado un test en la } \\
\text { web universitaria }\end{array}$ & $\begin{array}{l}\text { Alta.- Se sistematiza los } 3 \text { test, se } \\
\text { pueden acceder desde cualquier } \\
\text { navegador }\end{array}$ \\
\hline Disponibilidad & $\begin{array}{l}\text { Baja.- Se accede a los datos de forma } \\
\text { manual }\end{array}$ & $\begin{array}{l}\text { Alta.- Se accede a los datos de } \\
\text { forma digital }\end{array}$ \\
\hline Funcionabilidad & $\begin{array}{l}\text { Baja.- Tareas largas y tediosos causante de } \\
\text { bajo funcionamiento de registros y control }\end{array}$ & $\begin{array}{l}\text { Alta.- se optimiza las funciones al } \\
\text { ser digitales y los datos se generan } \\
\text { rápidamente }\end{array}$ \\
\hline Prevención de Errores & Baja.- Tabulación de información manual & $\begin{array}{l}\text { Alta.- ingreso correcto de varias } \\
\text { fichas automáticamente }\end{array}$ \\
\hline
\end{tabular}




\begin{tabular}{lll}
\hline Criterios & Antes & Ahora \\
\hline Tiempo de Búsqueda & Aproximadamente 30 a 45 minutos & $\begin{array}{l}\text { Aproximadamente } 20 \text { a } 60 \\
\text { segundos }\end{array}$ \\
$\begin{array}{l}\text { Tiempo de Ejecución } \\
\text { Tiempo de generación de } \\
\text { Reportes }\end{array}$ & $\begin{array}{l}\text { Aproximadamente } 45 \text { a } 60 \text { minutos } \\
\text { Aproximadamente } 30 \text { a } 45 \text { minutos }\end{array}$ & $\begin{array}{l}\text { Aproximadamente } 20 \text { a } 30 \text { minutos } \\
\text { Aproximadamente } 20 \text { a } 60\end{array}$ \\
\hline
\end{tabular}

Fuente: elaboración propia.

\section{Reflexiones finales}

Con el desarrollo de este artículo se consolidan los procesos de gestión que se lleva en la Dirección de Bienestar Estudiantil apoyado en una solución tecnológica, permite gestionar una gran cantidad de información de los estudiantes que ingresan por primera vez en la universidad y de esta manera procesarla para tomar decisiones oportuna y eficiente.

Se tomó en cuenta para el diseño del sistema propuesto nuevos estándares tecnológicos de desarrollo, de la misma forma las herramientas utilizadas cumplen los parámetros se ajustan por el departamento de sistema de la universidad ECOTEC, las cuales permitieron crear una aplicación dinámica y robusta, desarrollado para un ambiente web y que fácilmente es capaz de adaptarse a los cambios según las necesidades que tenga el usuario final.

Con la validación por la responsable del área de la aplicación de registro y control, manifestando en los resultados una reducción significativa en los tiempos de ingreso y salida de información del departamento de Bienestar Estudiantil, las tareas de orientación vocacional y psicológica en los procesos de captura de información y entrega de resultados disminuyeron en un $88 \%$.

\section{Bibliografía}

Alvia Choez, A. O., \& Vera Macías, W. A. (2018). Desarrollo de página web para la gestión de fichas estudiantiles para la universidad de guayaquil facultad de arquitectura y urbanismo carrera de arquitectura para la optimización de los procesos.

Amaya B., D. (2015). Metodologías ágiles en el desarrollo de aplicaciones para dispositivos móviles. Estado actual. Revista de Tecnología, 12(10.18270). doi:https://doi.org/10.18270/rt.v12i2.1291

Bravo C., J. (2011). Gestión de procesos (alineados a la estrategia). Santiago de Chile: Editorial Evolución S.A. Obtenido de http://www.evolucion.cl/resumenes/Resumen_libro_Gesti\%F3n_de_procesos_JBC_201 1.pdf

ECOTEC, U. (2016). Reglamento de Bienestar Estudiantil, Admisiones y Graduados. . Resolución Nro. 27-14-09-2016 CSU ECOTEC.

ECOTEC, U. (2017). Plan de Desarrollo Estratégico Institucional 2017 - 2021. Ecuador.

ECOTEC, U. (2019). Políticas Institucionales. Obtenido de https://www.ecotec.edu.ec/politicasinstitucionales/

García H., A., \& García P., F. (2015). Estudio Sobre La Evolución De Las Soluciones Tecnológicas Para Dar Soporte A La Gestión De La Información. Salamanca: Universidad de Salamanca. Obtenido de https://gredos.usal.es/jspui/bitstream/10366/125415/1/GRIALTR-2015-001.pdf 
Gómez O., R., Lona R., L., \& Jiménez S., L. (2016). Gestión de la innovación en instituciones de educación superior. Memoria del X Congreso de la Red Internacional de Investigadores en Competitividad.

Grande, M., Cañón, R., \& Cantón, I. (2016). Tecnologías de la información y la comunicación: Evolución del concepto y características. IJERI: International Journal of Educational Research and Innovation, 218-230. Obtenido de https://www.upo.es/revistas/index.php/IJERI/article/view/1703

Ley Orgánica De Educación Superior. (2010). Ecuador.

Mosqueda, H., \& Millán, J. (2013). Competitividad en empresas innovadoras de software y tecnologías informáticas. Cuadernos de Administración, 29(49), 37-44. Obtenido de http://www.scielo.org.co/pdf/cuadm/v29n49/v29n49a05.pdf

Posner, E. (1972). Archives in the Ancient World. Cambridge/ Massachusetts. Obtenido de http://files.archivists.org/pubs/free/ArchivesInTheAncientWorld-2003.pdf

Resolución Nro. 27-14-09-2016. (14 de Septiembre de 2016). Resolución Nro. 27-14-09-2016. Consejo Superior Universitario ECOTEC. Reglamento de Bienestar Estudiantil, Admisiones y Graduados. Samborondón, Guayas, Ecuador: Universidad ECOTEC.

Rodríguez S., O., \& Colina V., A. (2018). Propuesta tecnológica para la gestión eficiente del servicio médico de una universidad ecuatoriana. . Revista Espacios, 50. Obtenido de https://www.revistaespacios.com/a18v39n50/18395009.html

Salcedo-Muñoz, V., Quezada Abad, C., Núñez, L., Varela-Veliz, G., Novillo, E., \& Viteri, C. (2017). Bienestar estudiantil universitario en Ecuador: Caso unidades de bienestar estudiantil en las universidades de la provincia de El Oro. Revista Espacios. Obtenido de https://www.revistaespacios.com/a17v38n30/a17v38n30p17.pdf

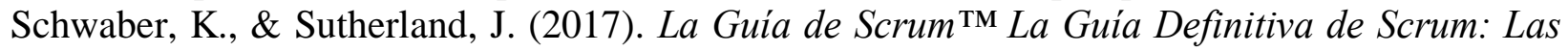
Reglas del Juego. Obtenido de https://www.scrumguides.org/docs/scrumguide/v2017/2017-Scrum-Guide-SpanishEuropean.pdf\#zoom $=100$

Solleiro, J. L., \& Castañón, R. (2016). Gestión Tecnológica Conceptos y prácticas. Obtenido de http://cambiotec.org.mx/site/wp-content/uploads/2017/09/E-Libro-Gestio\%CC\%81n2.pdf

Sommerville, I. (2011). Ingeniería del software. (Novena ed.). Mc Graw Hill.

Trigas G., M. (2012). Gestión De Proyectos Informáticos- Metodología Scrum. Barcelona: Universidad Abierta de Cataluña (UOC). Obtenido de http://openaccess.Uoc.Edu/Webapps/O2/Bitstream/10609/17885/1/Mtrigastfc0612mem oria.Pdf

UNAM. (2015). Metodologías y procesos de análisis de software. Obtenido de http://www.ptolomeo.unam.mx:8080/xmlui/bitstream/handle/132.248.52.100/175/A5\% 20Cap\%C3\%ADtulo\%202.pdf?sequence $=5$

Vázquez P., J., \& Ortiz M., V. (2018). Innovación educativa como elemento de la doble responsabilidad social de las universidades. IE Revista de investigación educativa de la REDIECH, 9(17), 133-144. Obtenido de http://www.scielo.org.mx/scielo.php? 